\title{
HEPATITIS B AND MALARIA AMONG NEPALESE BLOOD DONORS
}

\author{
Prakash Ghimire*, Bishnu Bhakta Dhungyel* and Bishnu Raj Tiwari* \\ ${ }^{*}$ Central Department of Microbiology,Tribhuvan University, Kirtipur, Kathmandu, Nepal.
}

\begin{abstract}
Viral hepatitis and malaria, both are the diseases with noticeable disease burden in Nepal. Malaria is seasonal with high disease burden during post rainy season. Severe malaria also shows similar symptoms to viral hepatitis. This is collaborative study has been conducted during June - September 2006 with the objective of determining the prevalence of hepatitis B and malaria in Nepalese blood donors to find out the need of routine malaria testing in each pint of donated blood.
\end{abstract}

During the study period, screening of malaria and hepatitis B surface antigen (HBsAg) were done in 1200 blood samples collected from blood donors at Kathmandu, Nepalguni and Biratnagar Blood Banks. Malaria diagnosis was done using one drop of blood from each unit of blood using Giemsa stained thick and thin smear microscopy, while hepatitis B surface antigen ( $\mathrm{HBsAg}$ ) was detected using commercial ELISA.

Of the total 1200 blood samples analyzed, 600 were collected at Kathmandu and 300 each at Nepalgunj and Biratnagar blood banks, situated in malaria endemic southern districts. Among the total 1200 samples, 1\% (12) of the sample was found reactive for $\mathrm{HBsAg}$; while only $0.33 \%$ (4) samples were positive for malarial parasite. $1.33 \%$ (8) samples from Kathmandu and $1.33 \%$ (4) samples from Nepalguni were positive for HBV. $1 \%$ (3) samples from Nepalgunj and $0.33 \%$ (1) sample from Biratnagar were found to be positive for malarial parasite. None of the samples from Biratnagar were positive for $\mathrm{HBsAg}$, while none of the samples from Kathmandu were positive for malarial parasite. All of the malaria cases were due to Plasmodium vivax. All the infected cases were male. Co-infection of malaria and HBV was not observed during the entire period of study.

However more extensive study is needed in other endemic areas of Nepal, the study indicated that the continuation of screening each point of donated blood for Hepatitis B, while screening of malarial parasites in donors of endemic terai districts may be useful in preventing transmission of malaria through transfusion.

Key words: Hepatitis; Malaria; ELISA; Blood donors; Blood transfusion.

\section{INTRODUCTION}

Malaria is both a modern and ancient plague. Although epidemics no longer occurs in temperate zones as a result of social and economic development, over 2000 million people, $41 \%$ of world's population still remain exposed to this disease. Three hundred to five hundred million cases occur each year worldwide affecting 90 countries or territories. Around 1.5 to 2.7 million deaths occurs each year due to malaria.

Nepal is more or less a rectangular area in between china (North) and India (South) with following east-west ranges and regions starting from north to south i. e. high Himalaya (23\%), Mountains (20\%) Midhills (30\%), Siwalik range area $(13 \%)$ and plain terai areas (14\%). Malaria situation in the country up to 1977 was maintained at the level between 10000 and 14000 cases annually. However from 1978 there was a real deterioration of situation in the country when malaria cases reached to 14212 from 11615 in 1977. With steady increase every year the number of cases reached to 16719 in
1983. There was a steep rise to 29188 cases in 1984, which again escalated to 42321 in 1985 with major outbreaks in Far western region, and minor ones in many parts of the country. The country has not been able to completely adopt the Global Malaria Control Strategy especially to carry out early diagnosis and prompt treatment of clinical malaria cases, to use personal protection and feasible bioenvironmental methods, and to implement strategy for prevention and control of epidemic outbreaks. Lack of laboratory facility especially in hard core, $P$. falciparum resistant and epidemic prone health post / sub health post and lack of regular monitoring of drug sensitivity status of P. falciparum are the major constraints of Malaria Control in Nepal. ${ }^{1}$

Hepatitis B virus (HBV) is one of the most important infectious agents causing acute and chronic morbidity worldwide. It is estimated that between 350 and 400 million people are chronic HBsAg carriers. ${ }^{2}$ According to the prevalence of HBsAg, the world can be divided into three areas of endemicity. Regions of the high endemicity, which include South East

Author for Correspondence: Prakash Ghimire, Central Department of Microbiology, Tribhuvan University, Kirtipur, Kathmandu, Nepal. 
Asia, Africa, China, the Artic Rim etc, have high prevalence of $8-20 \%$. Areas of intermediate endemicity have a prevalence of 2-7\% and include Eastern Europe and the Middle East, and low endemic areas such as Northern Europe, USA and Australia have prevalence below 2\%. ${ }^{3}$ According to World Health Report 2000, about 31000 deaths occur annually in South East Asia. ${ }^{4}$

Hepatitis B Virus is highly contagious and relatively easy to transmit from one infected individual to another, by bloodto-blood contact, during birth, unprotected sex, and by sharing needles, has relatively higher prevalence in the tropics. Thus there is the risk of acquiring HBV infection through blood transfusion. It is estimated that between 350 and 400 million people are chronic HBsAg carriers. ${ }^{5}$

In Nepal, hepatitis B is a major public health problem with carrier rate of 200 thousands and accounts for $6 \%$ of acute hepatitis. $1 \%$ of the populations are asymptomatic chronic hepatitis B surface antigen carriers, 39\% of patients suffer from chronic liver disease and $37 \%$ with hepatocellular carcinoma are HBsAg seropositive. ${ }^{5}$ Hepatitis B prevalence among the studied blood donors was $0.88 \%$ in total donors, more in replacement donors (1.37\%) as compare to volunteer donors $(0.79 \%)(\mathrm{P}<0.05)$. The seroprevalence of hepatitis $\mathrm{B}$ virus surface antigen (HBsAg) among the first time and repeat donors were $0.95 \%$ and $0.76 \%$ respectively $(\mathrm{P}>0.05){ }^{6}$

Blood transfusion is necessary to correct severe anemia, deficiency of plasma clotting factors, thrombocytopenia, immunodeficiency states and hypo-albuminaemia. Like all medical treatments, a blood transfusion should only be used when really necessary. The decision to give a blood transfusion to a patient is made only after careful consideration. Most people cope well with losing a moderate amount of blood. However, if larger amounts are lost, a blood transfusion is the best way of replacing the blood rapidly. Screening of blood is mandatory for providing safe blood. This includes blood grouping, ensuring compatibility of the donor and the recipient as well as testing for transfusiontransmitted agents. The transmission of infection through transfusion of infected blood and blood products can be easily prevented through screening of blood. ${ }^{7}$

Malaria and HBV are both blood borne diseases transmitted through transfusion of blood also. Both malaria and Hepatitis $B$ virus infection involves hepatomegaly and jaundice. Blood transfusion service in Nepal appears to be quite satisfactory. ${ }^{8}$ Mandatory screening of all donors for infections such as HIV, HBV, HCV and syphilis have significantly reduced the problem; but not completely eliminated such risk because the donor may be at the window period or lack sufficient response so that our tool can detect the response. The risk of such infection is clearly higher in recipients of blood obtained from commercial source as compared to blood from volunteer donors. In Nepal, Blood is screened for hepatitis, HIV and other STIs, but not for malaria. Therefore, the risk of acquiring malaria through transfusion is high in malaria endemic regions of Nepal.

Safe blood in adequate quantity has become all the more important due to increasing dangers of transmissible infections including HBsAg. ${ }^{9,10}$

Mandatory screening of all donors for such infections have significantly reduced the problem; but not completely eliminated such risk because the donor may be at the window period or lack sufficient response so that our tool can detect the response. The risk of such infection is clearly higher in recipients of blood obtained from commercial source such as compared to blood from volunteer donors. ${ }^{10,11}$

The study is aimed to find out the prevalence of malaria and hepatitis B infection among the blood donors of three different regions of Nepal viz. Central (Kathmandu), Eastern (Biratnagar) and Mid-western (Nepalgunj) where more than $75 \%$ of the blood is collected and circulated for transfusion. The findings of this study brings the first hand information regarding the risk of transfusion associated malaria and $\mathrm{HBV}$ infection which in turn would be an important information for the policy makers and the concerned authority for making appropriate screening policy of blood and blood products before transfusion.

\section{MATERIALS AND METHODS}

This is a collaborative study between Central Department of Microbiology, Tribhuvan University (CDM TU) and Nepal Red Cross Society Central Blood Transfusion Service (NRCS, CBTS). One of the authors was based full time at NRCS, CBTS during June - September 2006 and conducted the testing for academic thesis leading to Masters Degree in Microbiology, from Tribhuvan University; under the direct/indirect supervision of other authors for testing, recording and reporting. A total of 1200 blood samples were collected out of which 600 samples were collected from Kathmandu and 300 each from Nepalgunj and Biratnagar. Blood samples were collected by laboratory technicians and nurses following universal precautions and aseptic technique. Before collecting the sample, each donor was requested to fill the donor form (questionnaire).

Peripheral blood smears were prepared at the site of collections and transported to NRCS, CBTS; where microscopic examination was performed after Giemsa staining following standard protocols for the diagnosis of malaria. ${ }^{7}$

Blood/ Serum samples for presence of Hepatitis B surface antigen; were tested using the blood collected simultaneously in separate tubes during the blood collection in bags. Serum samples were separated from the clotted blood and evaluated for presence of HBsAg using commercial test kit protocol. ${ }^{12}$

All the test results were recorded in a register maintained at NRCS, CBTS, Kathmandu, maintaining the confidentiality of the test, ethically.

The data analysis was done as per the standard protocols ${ }^{13}$.

\section{RESULTS}

Out of the 1200 blood samples analyzed, 600 were collected from Kathmandu and 300 each were from Nepalgunj and Biratnagar blood banks. Among these, 94\% (1130) were of males and only $6 \%$ (70) were of female blood donors. 


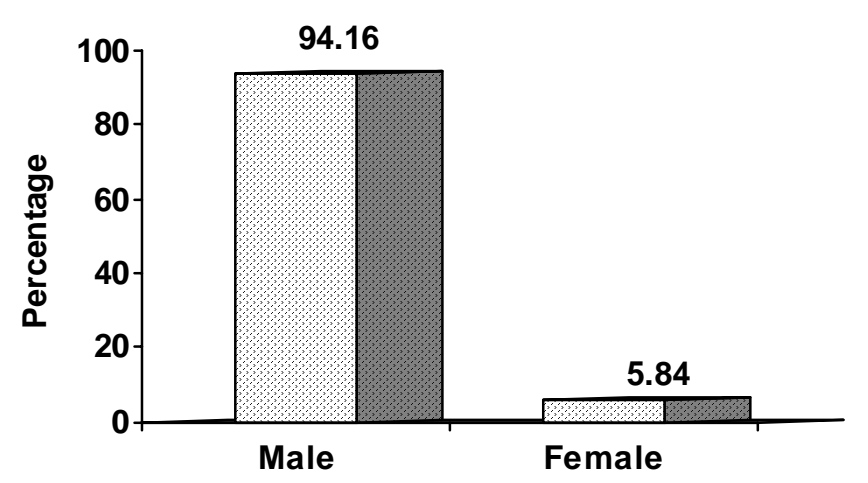

Figure 1: Gender-wise distribution of samples.

\section{Hepatitis B in Blood Donors}

Out of the 1200 blood samples studied, only 1\% (12) was found to be positive for HBsAg; of which. $0.75 \%$ (8) were from Kathmandu and $0.25 \%$ (4) from Nepalgunj. None of the donated blood samples studied from Biratnagar was positive for HBsAg.

Co-infection of malaria and Hepatitis B was not observed during the entire study of 1200 samples covering the major blood banks of the country.

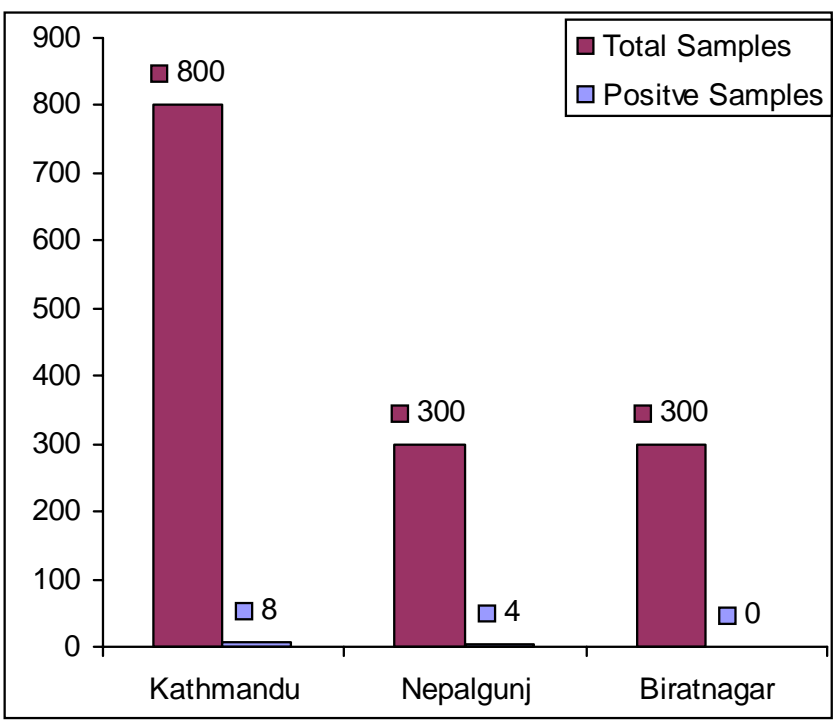

Figure 3: Hepatitis B in blood donors.

\section{Malaria in Blood Donors}

Among the 1200 samples analyzed in this study, 4 (0.33\%) were found to be positive for the malarial parasites while the remaining 1196 (99.67\%) samples were found to be negative. No smear samples collected from Kathmandu were found to be positive for malarial parasite. One smear sample collected from Biratnagar $(0.33 \%)$ and three samples collected from Nepalgunj (1\%) were positive for malarial parasite; however none of the samples from Kathmandu were positive for malarial parasite. All the malarial infections were due to Plasmodium vivax. (Fig. 2)

\section{DISCUSSION}

Screening of blood is now mandatory for many infectious diseases and is undertaken routinely in blood banks. Many studies have been done on effectiveness of screening tests

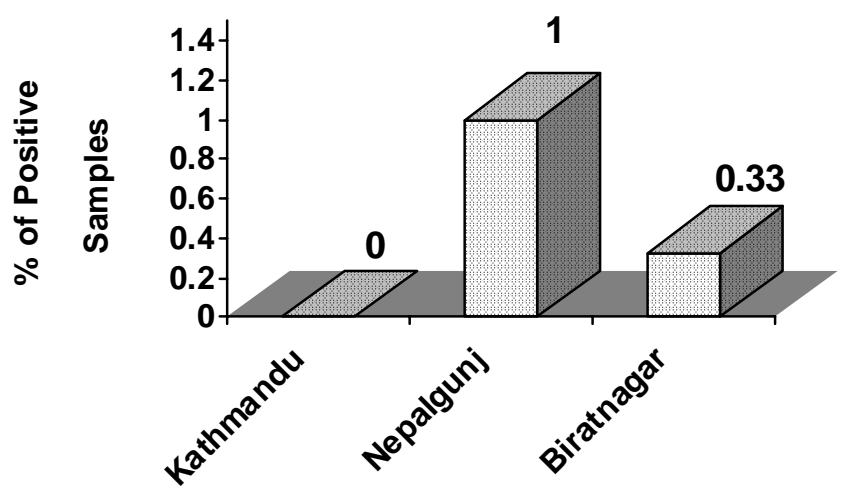

Figure 2: Malaria infection in donated blood.

for HIV, syphilis, HBsAg and HCV, But malaria was not included in the list. In view of malaria is endemic southern terai districts and malaria has been reported from 64 of 75 districts in Nepal, transfusion associated malaria is an issue to be addressed by blood donors/banks.

The blood banks, at present, do not routinely screen for malaria and no study in this regard has been so far undertaken in Nepal. There is no data on transfusion associated malaria in Nepal. Blood for transfusion is routinely screened for Hepatitis B. So this study was aimed at estimating the risk of malaria and Hepatitis B through blood transfusion and to find co-relation between these two diseases if any.

The result showed that the prevalence of infected donors is $0.33 \%$ for malaria and $1.00 \%$ for HBsAg. Highest prevalence of malaria was found in Nepalgunj (1\%) followed by Biratnagar $(0.33 \%)$. Since both of those regions lie in the malaria endemic area, Mandatory testing for malaria in such regions may be helpful in providing safe blood for transfusion. Similarly the prevalence of Hepatitis B was found to be same in the blood donors of Kathmandu and Nepalgunj (1.33\%).

In conclusion, the result of this small study raises an alarm to the existence of a significant risk of transfusion-transmitted malaria and Hepatitis B in Nepal. The results of this study represents just the tip of the iceberg, as this is just a representative study covering only 3 blood banks where as there are many blood banks in the endemic area which could not be included because of funding limitation. Thus the concerned authorities should take this issue seriously and must adopt measures to minimize this risk and require extensive study.

\section{ACKNOWLEDGEMENT}

We wish to express our sincere gratitude to all who directly or indirectly cooperated in accomplishment of such a study, including Dr. Manita Rajkarnikar, Director, NRCS, CBTS, Mr. Binod Kumar Mirdha, Mr. DP Upadhaya, and all the blood donors during the entire period of study.

\section{REFERENCES}

1 Ghimire, Prakash, Samantaray, J.C. and Mirdha, B.R. 2001. Comparitive evaluation of four different techniques used in the diagnosis of malaria. Journal of Institute of Medicine. 23:46-54.

2 Grosheide, P. and Van Damme, P. 1996. Prevention and Control 
of Hepatitis B in the Community. Communicable Diseases Series No.1. Antwerp: Viral Hepatitis Prevention Board, University of Antwerp.

3 Bonanni, P. 1998. Universal Hepatitis B Immunizaton: Infant, and Infant Plus Adolescents Immunization. Vaccine. 16:17-22.

4 Risbud, A., Mehendale, S., Basu, S., Kulkarni, S. and Walimbe, A. 2002. Prevalence and Incidence of Hepatitis B Virus Infection in STD Clinic Attendees in Pune, India. Sex Transm Infect. 78:169-173.

5 Shrestha, B. 2001. Seroprevalence of Hepatitis B Surface Antigen in Healthy Nepalese Male Population from Eastern Development Region. Journal of Institute of Medicine. 24:196-200.

6 Ghimire, Prakash, Thapa, Dinesh, Rajkarnikar, Manita and Tiwari, Bishnu Raj. 2006. HBsAg Sero-prevalence in Blood Donors of Kathmandu, Nepal Stupa. Journal of Health Science. 2(1\&2):24-26.

7 Ali, M.S., Gader, M., Kadaru, Y. and Mustafa, M.S. 2004. Screening Blood Donors for Malarial Parasite in Sudan. Ethiop. J. Health Development. 18:2.
8 JNMA (editorial). 2001. Possible Transmission of Blood Borne Infections in Medical and Dental Practice: Needs to be Tacked Seriously. Journal of Nepal Medical Association. 40:1.

9 Talib, V.H., Khurana, S.K., Verma, V.K., Ranga, S. 1996. Blood Transfusion Services: Blood Safety in India. Indian J Pathol Microbiol. 39:255-8.

10 Ghimire, Prakash, Thapa, Dinesh, Rajkarnikar, Manita and Tiwari, Bishnu Raj. 2006. HBsAg Seroprevalence in Blood Donors of Kathmandu, Nepal Stupa. Journal of Health Science. 2(1\&2):2426.

11 Walker, R.H. 1990. Technical Manual. 10th Edition. American Association of Blood Bank. pp 59-83.

12 Enzygonost, HBsAg ELISA kit insert, Dade Behring, Germany.

13 Mahajan, B.K. 1995. Biostatistiscs. BI Publication India. 